\title{
CAMBIOS EN EL ESPESOR DE LA CAPA DE FIBRAS NERVIOSAS TRAS CIRUGÍA DE CATARATAS MEDIDOS CON OCT: UN ESTUDIO PILOTO
}

\author{
RETINAL NERVE FIBER LAYER CHANGES AFTER CATARACT \\ SURGERY MEASURED BY OCT: A PILOT STUDY
}

PAREJA-ESTEBAN J ${ }^{1}$, TEUS-GUEZALA MA ${ }^{1}$, DRAKE-CASANOVA P², DAPENA-SEVILLA I ${ }^{2}$

\section{RESUMEN}

Objetivos: Analizar cambios en el espesor de la capa de fibras nerviosas de la retina (CFNR) peripapilar tras cirugía de cataratas.

Métodos: Medición del espesor medio de la CFNR de pacientes sometidos a cirugía de cataratas utilizando tomografía de coherencia óptica (OCT) III Stratus ${ }^{\circledR}$ antes de la cirugía, un día después de la intervención y transcurrido un mes. Los datos fueron analizados mediante el programa informático SPSS 12.0. Los resultados fueron comparados empleando el test pareado de dos colas t de Student.

Resultados: El grupo de estudio estaba compuesto de 74 ojos de 74 pacientes. El espesor medio de la CFNR antes de la cirugía de cataratas (AVGT0) fue 90,71 um (DE: 19,93), el día después de la cirugía (AVGT1) fue 88,30 $\mu \mathrm{m}$ (DE: 20,59); y un mes después (AVGT2) 97,45 um (DE: 14,30). No se encontraron diferencias estadísticamente significativas entre AVGT0 y AVGT1 $(\mathrm{P}=0,37)$; sin embargo, dicha diferencia sí se encontró entre AVGT1 y AVGT2 $(\mathrm{P}=0,002)$. También se encontró diferencia

\begin{abstract}
Purpose: To analyze changes in peripapillary retinal nerve fiber layer (RNFL) after cataract surgery. Methods: The average RFNL thickness of patients who underwent cataract surgery was measured using the optical coherence tomography (OCT) III Stratus ${ }^{\circledR}$ before surgery, the day after and one month later. The data were analyzed using the SPSS 12.0 software. We used paired two tail student's $t$ test for comparisons.

Results: The study group was composed by 74 eyes of 74 patients. The RNFL thickness average was $90.71 \mu \mathrm{m}$ (SD: 19.93), 88.30 $\mu \mathrm{m}$ (SD: 20.59) and $97.45 \mu \mathrm{m}$ (SD: 14.30), before cataract surgery (AVGT0), the day after surgery (AVGT1) and one month later (AVGT2) respectively. We did not find statistically significant difference between AVGT0 and AVGT1 $(\mathrm{P}=0.37)$; however, statistically significant difference between AVGT1 and AVGT2 (P= $0.002)$ as well as in the image signal quality between AVGT0 and AVGT2 (P=0.0001).

Conclusions: The RNFL average thickness measured by OCT III Stratus ${ }^{\circledR}$ showed an increase one
\end{abstract}

\footnotetext{
Recibido: 17/5/07. Aceptado: 17/6/09.

Servicio de Oftalmología. Hospital Universitario Príncipe de Asturia. Alcalá de Henares. Madrid. España.

1 Doctor en Medicina.

2 Licenciado en Medicina.

Correspondencia:

Jesús Pareja Esteban

Servicio de Oftalmología. Hospital Universitario Príncipe de Asturias.

Campus Universitario. Carretera Alcalá-Meco, s/n

28805 Alcalá de Henares (Madrid)

España

E-mail: jeparejaes@yahoo.es
} 
estadísticamente significativa en la calidad de señal de la señal entre AVGT0 y AVGT2 ( $\mathrm{P}=0,0001)$.

Conclusiones: El espesor medio de la capa de fibras nerviosas experimenta un incremento cuando es medido mediante OCT III Stratus ${ }^{\circledR}$ un mes tras la cirugía de cataratas. Este fenómeno se acompaña de una mejoría en la calidad de la señal.

Palabras clave: Tomografía de Coherencia Óptica, cirugía de cataratas, nervio óptico, estudios de seguimiento, glaucoma. month after cataract surgery. This phenomenon is accompanied with an improvement in signal quality (Arch Soc Esp Oftalmol 2009; 84: 305-310).

Key words: Optical Coherence Tomography, cataract surgery, optic nerve; follow-up studies, glaucoma.

\section{INTRODUCCIÓN}

La cirugía de cataratas mediante facoemulsificación sigue siendo hoy en día uno de los procedimientos quirúrgicos más habituales en la práctica oftalmológica actual. Gracias a la introducción de nuevas técnicas de imagen retiniana, como la Tomografía de Coherencia Óptica (OCT), es posible realizar un estudio preciso con alta resolución de los cambios que se suceden a nivel del espesor medio de la capa retiniana de fibras nerviosas (CNFR) peripapilar tras la cirugía ocular, incluso cuando dichos cambios son sutiles (1).

La presencia de cataratas y de alteraciones que modifican la transparencia de los medios oculares dificulta la visualización del polo posterior, favoreciendo que las imágenes de retina que se obtienen mediante el empleo de OCT tengan una pobre calidad. Este hecho puede conducir hacia una falsa medición del espesor de capa de fibras nerviosas, resultando ésta más delgada.

Con frecuencia encontramos en un mismo paciente la asociación de cataratas con patologías del nervio óptico como el glaucoma, en el que la exploración del espesor de la CFNR puede tener cierta relevancia no solo en el diagnóstico sino también en el seguimiento de dichos pacientes.

Por ello planteamos el diseño de este estudio piloto en el cual estudiamos las implicaciones que la cirugía de cataratas puede tener en la exploración del espesor de la CFNR.

\section{SUJETOS, MATERIAL Y METODOS}

Se estudiaron 74 ojos de 74 pacientes que iban a ser sometidos a cirugía de cataratas, previo consen- timiento informado tanto para dicha intervención como para la obtención de las imágenes con OCT.

Se incluyeron aquellos pacientes que no presentaban patología ocular glaucomatosa ni otras alteraciones en la transparencia de medios. De la misma manera, se eligieron pacientes con un rango de presión intraocular (PIO) por debajo de $21 \mathrm{mmHg}$.

El procedimiento quirúrgico fue llevado a cabo por un mismo cirujano (M.A.T.) mediante técnica de facoemulsificación e implante de lente intraocular (LIO) de cámara posterior. Se empleó anestesia tópica. Las incisiones se realizaron en córnea clara. Se eliminaron todos aquellos casos en los que se apreciaron complicaciones quirúrgicas a corto o medio plazo.

Las mediciones fueron realizadas por un mismo observador (J.P.E.). Se utilizó OCT III Stratus ${ }^{\circledR}$ (Carl Zeiss Meditec), realizándose todas las mediciones bajo las mismas condiciones de midriasis. Se realizaron tres exploraciones: antes de la cirugía, el día después de la misma y tras un mes. En cada una de las exploraciones, se realizaron tres mediciones, eligiéndose para el análisis aquella que presentaba mejor señal de calidad de imagen.

En cada una de las mediciones se obtuvo una imagen del área peripapilar en un diámetro de $3,4 \mathrm{~mm}$, para ello se empleó el programa «Fast RNFL Thickness» de OCT III Stratus ${ }^{\circledR}$. Con el fin de conseguir el perfecto centrado del scanner, se empleó en todos los casos fijación interna.

Se extrajeron los datos de espesor medio de RFNL utilizando el software de análisis del mismo instrumento.

Los resultados fueron analizados estadísticamente con el programa informático de estadística SPSS 12.0 para Windows (SPSS Inc., Chicago, Illinois, USA). Se aplicó el test de la «t de Student» para datos pareados considerándose estadísticamente significativos aquellos resultados con $\mathrm{p}<0,05$. 


\section{RESULTADOS}

El grupo de estudio estuvo compuesto por 74 ojos (38 derechos; 36 izquierdos) de 74 pacientes (32 hombres, 42 mujeres), con una edad media de 71,62 (DE: 9,46) años.

El espesor medio peripapilar de CFNR antes de la cirugía de cataratas (AVGT0) fue 90,71 (DE: 19,93) um; el día después de la cirugía (AVGT1) fue 88,30 (DE: 20,59) um; y un mes después (AVGT2) fue de 97,45 (DE: 14,30) $\mu \mathrm{m}$.

No se encontraron diferencias estadísticamente significativas $(\mathrm{p}>0,05)$ entre AVGT0 y AVGT1; sin embargo, dicha diferencia si se apreció entre AVGT1 y AVGT2 $(p=0,002)$ con un mayor espesor medio de la CFNR un mes después de la cirugía de cataratas.

Al analizar el comportamiento de la CFNR según sectores peripapilares, resultan especialmente interesantes los resultados obtenidos en los sectores superior e inferior (tablas I y II).

En cuanto a la calidad de imagen que ofrece OCT III Stratus ${ }^{\circledR}$ (Signal) los resultados fueron de 4,19 (DE: 1,92) en las mediciones AVGT0, 4,84 (DE: 2,29) en AVGT1 y 4,92 (DE: 1,55) en el caso de AVGT2. No se apreciaron diferencias con significación estadística entre la calidad de las imágenes tomadas en AVGT0 y AVGT1, aunque dichas diferencias si se encontraron al comparar AVGT0 con AVGT2 $(\mathrm{p}=0,001)$.

\section{DISCUSIÓN}

La OCT es una prueba no invasiva que ha demostrado ser de gran utilidad en el estudio de patología macular con una alta precisión, ya que la resolución de imágenes puede llegar a ser hasta de $10 \mu \mathrm{m}$; del mismo modo ha demostrado su utilidad en el diagnóstico y seguimiento de patología del nervio óptico.

Sin embargo, no son del todo bien conocidos los cambios estructurales que se suceden a nivel del polo posterior tras la realización de cirugías tan rutinarias como la facoemulsificación del cristalino, a cuyo conocimiento está contribuyendo el análisis con OCT.

Algunos autores como Ching H-Y et al (1) han encontrado diferencias estadísticamente significativas consistentes en un mayor grosor foveal y espesor retiniano central preoperatorio al compararlo con las semanas 2 y 8 del postoperatorio de la cirugía de cataratas, no manteniéndose esta tendencia en cuanto a espesor central retiniano en la semana cuarta.

De la misma manera, Biro $\mathrm{Z}$ et al. (2) no encuentran un incremento de espesor retiniano significativo ni perifoveolar ni foveolar el día después de la cirugía. Sin embargo 30 días después de la intervención encuentran un aumento significativo.

Grewing y Becker (3) demostraron que no existían diferencias entre la medición preoperatoria del gro-

Tabla I. Comportamiento de espesor de la CFNR peripapilar en sector Superior

\begin{tabular}{|c|c|c|c|}
\hline & Espesor medio & Comparaciones & Significación estadística (P) \\
\hline SA0 & $105,07 \mathrm{DE} 27,15 \mu \mathrm{m}$ & SA0 Vs. SA1 & $P=0,045$ \\
\hline SA1 & 111,71 DE $29,33 \mu \mathrm{m}$ & SA1 Vs. SA2 & $\mathrm{P}=0,029$ \\
\hline SA2 & $116,59 \mathrm{DE} 22,07 \mu \mathrm{m}$ & SA0 Vs. SA2 & $\mathrm{P}=0,001$ \\
\hline
\end{tabular}

CFNR: Capa de fibras nerviosas de la retina; SA0: espesor CFNR superior preoperatorio; SA1: espesor CFNR superior 24 horas después de la cirugía de cataratas; SA2: espesor CFNR superior 1 mes después de la cirugía de cataratas.

Tabla II. Comportamiento de espesor de la CFNR peripapilar en sector Inferior

\begin{tabular}{llcc}
\hline & Espesor medio & Comparaciones & Significación estadística (P) \\
\hline IA0 & $119,50 \mathrm{DE} 27,82 \mu \mathrm{m}$ & IA0 Vs. IA1 & $\mathrm{P}=0,016$ \\
IA1 & $111,06 \mathrm{DE} 31,71 \mu \mathrm{m}$ & IA1 Vs. IA2 & $\mathrm{P}=0,009$ \\
IA2 & $122,63 \mathrm{DE} 24,18 \mu \mathrm{m}$ & IA0 Vs. IA2 & $\mathrm{P}=0,047$ \\
\hline \hline
\end{tabular}

CFNR: capa de fibras nerviosas de la retina; IA0: espesor CFNR superior preoperatorio; IA1: espesor CFNR superior 24 horas después de la cirugía de cataratas; IA2: espesor CFNR superior 1 mes después de la cirugía de cataratas. 
sor macular retiniano y 30 minutos después de la cirugía de cataratas.

En cuanto al espesor medio de CFNR peripapilar, El-Ashry et al (4) han hallado significación estadística a favor de un mayor espesor medio de CFNR tras la realización de cirugía de cataratas en 24 pacientes, un mes después de la intervención. Por otro lado, encontraron una mejor calidad de señal transcurrido un mes desde la cirugía.

El hallazgo de una correlación positiva entre el aumento de la señal medido según el parámetro «Signal Strength» y el incremento del grosor de la CFNR ha sido constatado igualmente por diversos autores recientemente (8-10).

Estos resultados, son acordes con los obtenidos en nuestro estudio, si bien aportamos además la evidencia de que dichos cambios de espesor a nivel peripapilar no son detectables tras 24 horas de la cirugía. Quizás sea necesario un tiempo mayor a 24 horas desde la cirugía para que los factores implicados en la génesis de dicho incremento de capa de fibras nerviosas produzcan tal efecto (5).

Sin embargo al analizar los sectores peripapilares superior e inferior, obtenemos resultados estadísticamente significativos desde las primeras 24 horas. En el caso del sector superior se aprecia un mayor espesor postquirúrgico inmediato, mientras que el sector inferior experimenta una disminución de espesor.

Un mes tras la cirugía se mantienen las diferencias a favor de un mayor espesor en ambos sectores que en la determinación basal. No obstante, analizando el sector inferior encontramos una débil significación estadística $(\mathrm{p}=0,047)$, lo que puede indicar que si bien se produce un incremento en el espesor de capa de fibras inferiores, las diferencias pre y postquirúrgicas en este sector son mínimas convirtiéndolo en un parámetro menos dependiente de la calidad de imagen, y por lo tanto de una fiabilidad mayor en el estudio del nervio óptico de pacientes con catarata. Resultan especialmente interesantes estos resultados si tenemos en cuenta la utilidad del estudio de ambos sectores en el diagnóstico de glaucoma mediante OCT (11).

El aumento en el espesor medio de CFNR peripapilar después de un mes postoperatorio, se acompaña además de una mejoría en la calidad de la señal.

Este resultado puede ser explicado por la disminución del edema corneal e inflamación corneal que acontecen en las primeras 24 horas del postoperatorio, así como por la eliminación del propio cristalino que puede influir en la obtención de imágenes $(6,7)$.
Las condiciones más favorables de transparencia de medios así como de ausencia de inflamación corneal un mes después de la cirugía pueden ser la causa de que la exploración del espesor de CFNR en este momento sea la que más se aproxime a la realidad, y que por lo tanto bajos niveles de Signal Strength supongan un artefacto en la medición de la capa de fibras nerviosas.

Por otro lado resultaría interesante estudiar otros factores que puedan influir en que la capa de fibras nerviosas experimente un incremento tras la cirugía, como por ejemplo la inflamación intraocular postquirúrgica. En ese sentido estudios de un mayor tiempo de evolución en los que se monitorice el comportamiento de CFNR podrán aportar más información, partiendo de la hipótesis que dicha inflamación sería menor en el tiempo, y por tanto la capa de fibras mostraría un menor grosor. En este caso se deberían controlar factores que afectasen dicha señal como por ejemplo la opacificación de la cápsula posterior.

Por lo tanto, debe tenerse en consideración los efectos que conlleva la presencia de catarata tanto en aquellos estudios en los que se evalúen los cambios en espesor de capa de fibras nerviosas mediante OCT, como aquellos en los que se interpretan resultados de edema macular cistoide después de la cirugía de cataratas.

En la práctica clínica, los cambios obtenidos en la medición de CFNR como consecuencia de la cirugía de catarata, deben ser tenidos en cuenta en aquellos pacientes en los que OCT supone una herramienta para el seguimiento de sus patologías oculares de base.

Sin embargo, estos resultados quedan sujetos a estudios con bases muestrales mayores, en los que puedan corroborarse de forma más fehaciente las diferencias estadísticas halladas. Cabe destacar así mismo como limitación del presente estudio la reducida calidad de las imágenes obtenidas, consecuencia primera de la presencia de cataratas.

\section{BIBLIOGRAFÍA}

1. Ching HY, Wong AC, Wong CC, Woo DC, Chan CW. Cystoid macular oedema and changes in retinal thickness after phacoemulsification with optical coherence tomography. Eye 2006; 20: 297-303.

2. Biro Z, Balla Z, Kovacs B. Change of foveal and perifoveal thickness measured y OCT after phacoemulsification and IOL implantation. Eye 2008; 22: 8-12. 
3. Grewing $R$, Becher $H$. Retinal thickness inmediately after catartact surgery measured by optical coherence tomography. Ophthalmic Surg Lasers 2000; 31: 215 217.

4. El-Ashry M, Appaswamy S, Deokule S, Pagliarini S. The effect of phacoemulsification cataract surgery on the measurement of retinal nerve fiber layer thickness using optical coherence tomography. Curr Eye Res 2006; 31: 409413.

5. Hee MR, Puliafito CA, Wong C, Ducker JS, Reichel E, Rutledge B, et al. Quantitative assessment of macular edema with optical coherence tomography. Arch Ophthalmol 1995; 113: 1019-1029.

6. Sourdille P, Santiago PY. Optical coherence tomography of macular thickness after cataract surgery. J Cataract Refract Surg 1999; 25: 256-261.

7. Van Velthoven $M E$, van der Linden $M H$, de Smet $M D$, Faber DJ, Verbraak FD. Influence of cataract on optical coherence tomography image quality and retinal thickness. Br J Ophthalmol 2006; 90: 1259-1262.

8. Cheung CY, Leung CK, Lin D, Pang CP, Lam DS. Relationship between retinal nerve fiber layer measurement and signal strength in optical coherence tomography. Ophthalmology 2008; 115: 1347-1351.

9. Wu Z, Vazeen M, Varma R, Chopra V, Walsh AC, LaBree $L D$, et al. Factors associated with variability in retinal nerve fiber layer thickness measurements obtained by optical coherence tomography. Ophthalmology 2007; 114: 1505-1512.

10. Savini G, Zanini M, Barboni P. Influence of pupil size and cataract on retinal nerve fiber layer thickness measurements by Stratus OCT. J Glaucoma 2006; 15: 336-340.

11. Gyatsho J, Kaushik S, Gupta A, Pandav SS, Ram J. Retinal nerve fiber layer thickness in normal, ocular hypertensive, and glaucomatous Indian eyes: an optical coherence tomography study. J Glaucoma 2008; 17: 122-127. 
